\title{
Effectiveness of Genicular Nerve Cooled Radiofrequency Ablation on Chronic Knee Osteoarthritis Pain
}

\author{
Jonathan Carrier, DO'1, Michelle Poliak-Tunis, MD², and Scott Hetzel, MS ${ }^{3}$
}

Background: Outside of an invasive total knee arthroplasty, the available therapies for the treatment of pain secondary to knee osteoarthritis (OA) provide marginal and short-lived symptomatic relief. Genicular nerve radiofrequency ablation (RFA) serves as an alternative treatment modality for OA-associated knee pain and disability.

Objectives: To quantify the effectiveness of cooled radiofrequency ablation (C-RFA) of the genicular nerves for chronic knee pain secondary to $\mathrm{OA}$.

Study Design: Retrospective chart review performed using Redcap, implementing current procedural terminology codes.

Setting: An academic pain management center. Methods: Study population included patients treated with C-RFA from April 2015 through June 2017. Numeric Rating Scale (NRS) data were analyzed at 3 time points: 2 weeks, 4-6 weeks, and 7-33 weeks post-RFA (extended follow-up). Primary outcome for statistical analysis was NRS and the change in NRS from baseline at each of the 3 predetermined time points. Differences between the change in NRS and the number of diagnostic blocks performed (1 vs. 2) was evaluated. Correlation between the change in NRS and patient body mass index (BMI) was calculated.

Results: Pre-RFA average NRS scores were available for 47 knees from 31 individuals, which were included in the analysis. The mean NRS score decreased by $50 \%$ at 2 weeks $(\mathrm{n}=33 ; P$ $<0.001), 55 \%$ at $4-6$ weeks $(\mathrm{n}=18 ; P<0.001)$, and $26 \%$ at $7-33$ weeks $(\mathrm{n}=18 ; P=0.009)$.
Eight patients (12 knees) provided specific data on the total duration of relief following RFA. The mean duration was 39 weeks or approximately 9 months. There were no statistically significant differences between groups receiving 1 versus 2 diagnostic blocks at 2 weeks or 4-6 weeks postRFA. At 7-33 weeks, those who received 1 block had a decrease in NRS of -3.1 , whereas those who received 2 blocks had an increase in NRS of $+0.1(P=0.008)$. There was no correlation identified between BMI and change in NRS at any time point.

Limitations: This study's retrospective design inherently leads to a higher risk of selection bias. The sample size was relatively small as a high percentage of patients were lost to follow-up. The primary outcome measure for this study was the change in mean NRS pain score, and the mean of ordinal data with a nonnormal distribution lacks validity in statistical analysis.

Conclusions: In this study population, C-RFA of the genicular nerves lead to $50 \%$ or greater pain relief at 2 weeks and 4-6 weeks postintervention. A $26 \%$ pain relief was achieved at 7-33 weeks, but this did not meet the established minimal clinically important difference cutoff. Two diagnostic genicular nerve blocks did not improve the rate of treatment success when compared to a single diagnostic block. BMI does not appear to correlate with outcomes.

Key words: Genicular radiofrequency ablation, genicular RFA, cooled radiofrequency ablation, chronic knee pain, knee osteoarthritis

From : ${ }^{1}$ Physical Medicine and Rehabilitation, Washington University, St. Louis, MO; ${ }^{2}$ University of Wisconsin School of Medicine and Public Health, Madison, WI; ${ }^{3}$ University of Wisconsin, Madison, WI

Author for correspondence: Jonathan Carrier, DO

Address: Physical Medicine and Rehabilitation, Washington University in St. Louis, 660 South Euclid Ave., Campus Box 8233, St. Louis, MO 63110 E-mail: jcarrier@wustl.edu 
Osteoarthritis (OA) is among the most common diagnoses encountered in clinical practice. OA is a heterogeneous disorder involving the progressive loss of articular cartilage, development of subchondral sclerosis, and approximation of joint margins $(1,2)$. OA affects an estimated $21 \%-36.8 \%$ of US adults (3-5). In a recent systematic review, hip and knee OA were ranked as the 11 th contributor to global disability out of 291 diseases across 187 countries (6). Over the past 20 years, clinicians and researchers have observed significant increases in the prevalence of symptomatic knee OA in the United States, independent of age and body mass index (BMI) (7). The number of total knee replacements performed annually has similarly increased, more than doubling from 1999 to 2008 (8). Treatment efforts routinely focus on alleviating pain and stiffness associated with the disorder, secondary to underlying inflammation and effusion. Addressing modifiable risk factors such as BMI, comorbid conditions, knee re-injury, and pain coping mechanisms are also important considerations (9).

Current symptom management strategies include pharmacological therapies, bracing, wedging, physical therapy, and intraarticular joint injections. Proposed disease modifying agents for the treatment of OA have shown variable effectiveness. A metaanalysis by Gallagher et al (10) suggested that the long-term use of glucosamine and chondroitin sulfate supplementation may have small but significant chondroprotective effects. The same analysis revealed no discernible effect on the progression of OA with nonsteroidal anti-inflammatory drugs, soy unsaponifiables, or hyaluronic acid (HA)-based injections (10). Nonetheless, intraarticular HA and corticosteroid (CS) injections remain first-line therapies for symptomatic knee OA. The analgesic effect of CS is generally short-lived, and typically most pronounced within the first month postinjection. The treatment effect of HA, however, may last in upward of 6 or more months (11). A recent randomized controlled trial by McAlindon et al (12) revealed significant cartilage volume loss following a 2-year course of CS injections when compared with saline solution. There were no significant differences between the CS and saline solution groups in Western Ontario and McMaster Universities Osteoarthritis Index pain, function, or stiffness subscales (12). There is limited to moderate evidence available supporting the potential efficacy of intraarticular platelet rich plasma injections for the treatment of OA (13). Outside of an invasive total knee arthroplasty (TKA), available therapies for the treatment of pain secondary to knee OA provide marginal and short-lived symptom relief.

Genicular nerve radiofrequency ablation (RFA) serves as an alternative treatment modality for OAassociated knee pain and disability. The procedure was initially implemented for patients who were nonresponsive to conservative therapy, were poor surgical candidates, or who wanted to avoid TKA (14). The genicular nerves are articular branches supplied by larger parent nerves that include the femoral, common peroneal, saphenous, and obturator nerves. Cadaveric dissections reveal that 6 nerves supply the joint capsule. The distal branches of these nerves have consistent localization near the joint. Four of these branches are accessible via percutaneous ablation, including the middle, superior lateral, superior medial, and inferior medial genicular nerves. RFA implements a high-frequency current at an electrode tip, inducing ionic vibrations, which results in friction and the generation of heat. This heat causes thermocoagulation of local neuronal tissue, resulting in distal Wallerian degeneration. The radiofrequency lesion is believed to halt nociceptive afferent input from the genicular nerves innervating the anterior joint capsule (15). Choi et al (14) performed one of the earliest randomized controlled trials, resulting in 12 weeks of $>50 \%$ knee pain relief in 10 out of 17 patients. Our current study adds to the growing body of literature on the therapeutic effects of genicular RFA for the management of chronic knee pain secondary to $O A$. Current data regarding the effectiveness of cooled radiofrequency ablation (C-RFA) remains limited. The study aimed to quantify the treatment effect of genicular C-RFA on chronic knee pain, and potentially establish the duration of effect. Secondarily, this study aimed to determine whether or not the number of preRFA diagnostic blocks or patient BMI had an effect on the outcomes of the procedure.

\section{METHODS}

The study design was a retrospective chart review conducted at an academic pain management center. The study population included patients treated with 

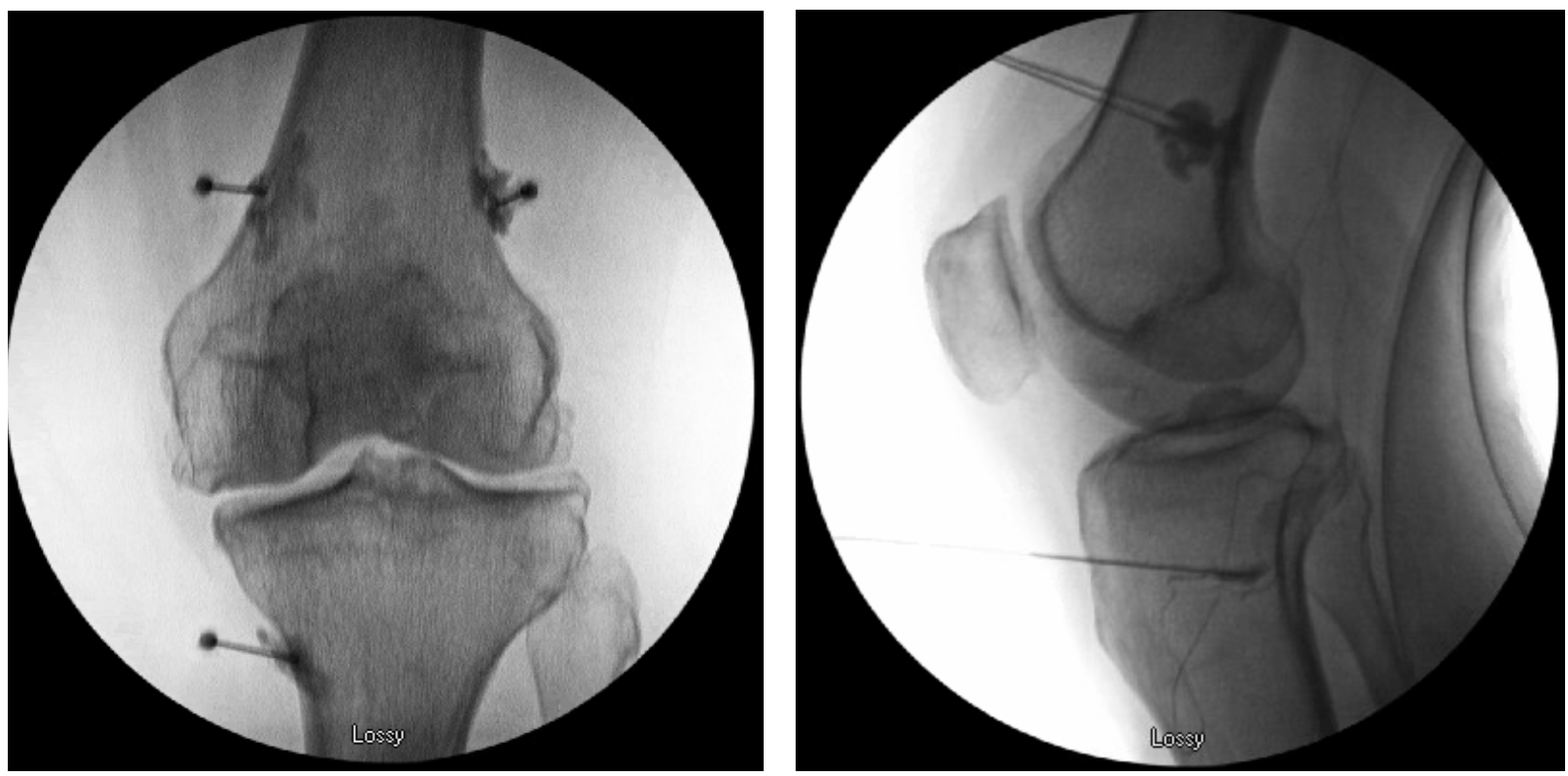

Fig. 1. Coronal (A) and sagittal (B) fluoroscopic images of the final needle positions for diagnostic blocking of the superomedial, superolateral, and inferomedial genicular nerves with appropriate contrast medium spread.

genicular nerve RFA from April 2015 through June 2017, identified within the electronic medical record (EMR). The clinicians used REDCap (Vanderbilt, Nashville, TN) to perform the retrospective chart review, implementing current procedural terminology codes. To qualify for genicular RFA at this institution, patients must have chronic knee pain in the setting of OA. Chronic knee pain was defined as pain lasting $>6$ weeks. These patients also had successful diagnostic genicular nerve anesthetic blockade, defined as $\geq$ $50 \%$ reduction in concordant pain. Exclusion criteria included the following: BMI > 50, active infection being treated with antibiotics, current use of anticoagulants (not held preprocedurally), and an inability to sign informed consent. Patients underwent 1 or 2 diagnostic genicular nerve blocks to be considered a candidate for C-RFA. Both blocks and C-RFA were performed with the patient in the supine position, with a bolster placed behind the knee producing 20-30 degrees of knee flexion. Under fluoroscopic guidance, clinicians directed a 22-guage, 2.5-inch spinal needle toward the anatomic localization of the superior medial, superior lateral, and inferior medial genicular nerves. The superior medial genicular nerve is located at the junction of the medial femoral shaft and femoral condyle (coronal plane). The superior lateral genicular nerve is located at the junction of the lateral femoral shaft and femoral condyle (coronal plane). The inferior genicular nerve is located at the junction between the medial tibial shaft and tibial condyle (coronal plane). Each genicular nerve is located at the midpoint of the diaphysis (sagittal plane). Once the target was reached on anteroposterior and lateral views, iohexol $(0.3 \mathrm{~mL})$ was injected, demonstrating appropriate contrast medium spread (Fig. 1). Lidocaine $(2 \%, 0.5$ $\mathrm{mL}$ ) was then injected at each site and the needles were removed.

During the first year in which this procedure was performed at this institution (April 2015 to April 2016), a single diagnostic block was administered prior to RFA. Following this, the protocol was changed to 2 diagnostic blocks prior to RFA to theoretically remove placebo responders. Those that underwent a second diagnostic nerve block received bupivacaine $(0.5 \%$, $0.5 \mathrm{~mL}$ ) at each injection site as opposed to lidocaine. After completion of the nerve block(s) with successful relief of concordant pain, C-RFA was performed. CRFA was implemented owing to its ability to create a larger lesion size when compared with conventional 

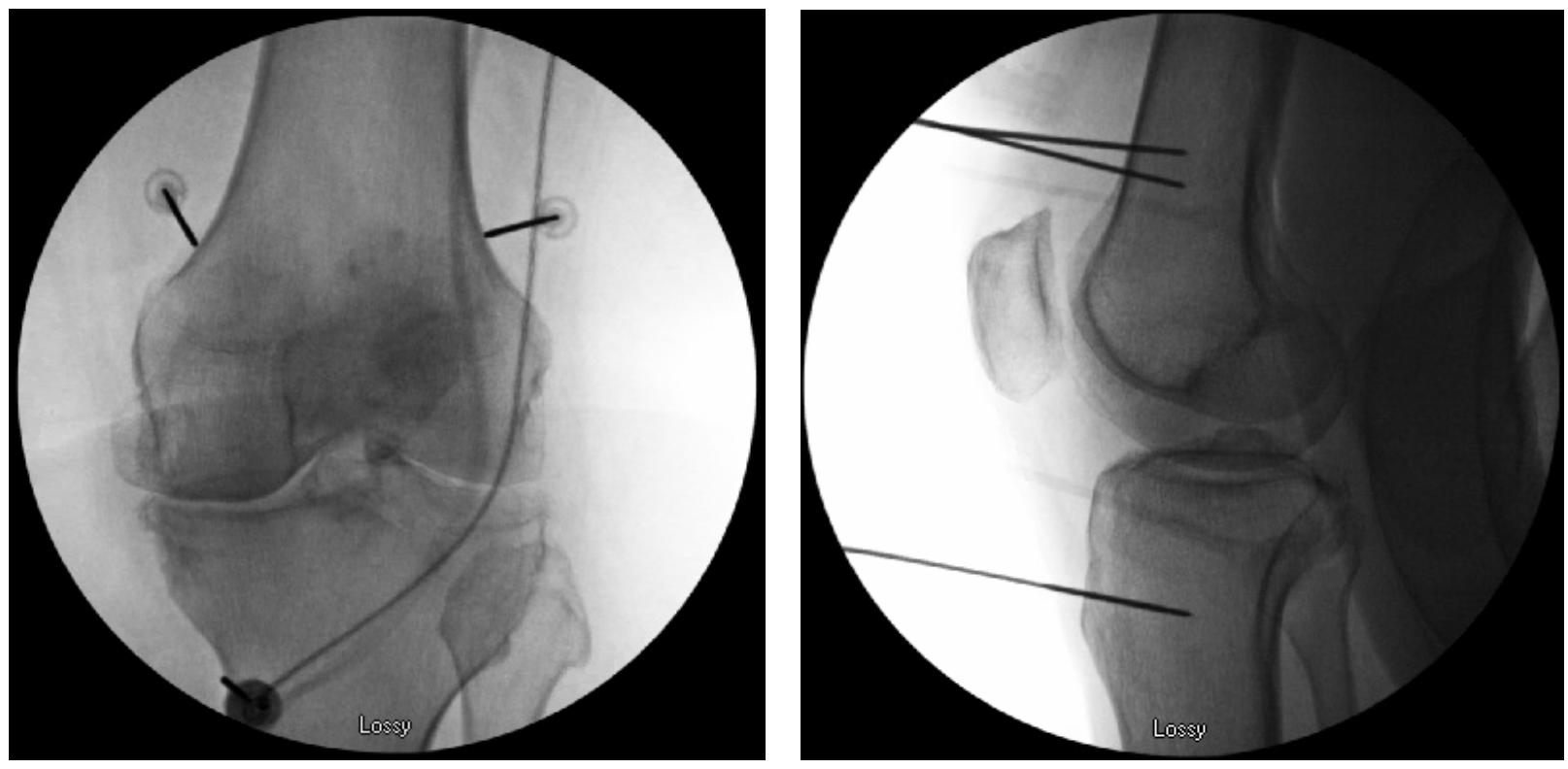

Fig. 2. Coronal (A) and sagittal (B) fluoroscopic images of the final needle positions for C-RFA of the superomedial, superolateral, and inferomedial genicular nerves.

RFA, increasing the probability of denervation (16). Probes with $4 \mathrm{~mm}$ active tips were directed toward the superior medial, superior lateral, and inferior medial genicular nerves (Fig. 2). The probes were advanced using a tunnel technique until bony contact was achieved with probe tips localized at the midpoint of the diaphysis on true lateral views. A total of $1.5 \mathrm{~mL}$ of equal volumes of $2 \%$ lidocaine and $0.5 \%$ bupivacaine were instilled at each site prior to RF lesioning. One lesion was created at each site at a temperature of $60^{\circ} \mathrm{C}$ for 2 minutes and 30 seconds. This was followed by instillation of $1.5 \mathrm{~mL}$ of equal volumes of $2 \%$ lidocaine, $0.5 \%$ bupivacaine, and dexamethasone $(10 \mathrm{mg} / \mathrm{mL})$ at each site. Dexamethasone was used empirically to decrease the theoretical risk of postRFA neuritis (17).

Patient characteristic data included gender, BMI, laterality (left vs. right), number of blocks performed prior to RFA, history of knee surgery, and history of prior intraarticular injections. Numeric Rating Scale (NRS) data were analyzed at 3 time points: 2 weeks, 4-6 weeks, and 7-33 weeks post-RFA (extended followup). At 2 weeks, patients were required to mail in a pain questionnaire. At the 4-6 week mark, data were gathered on patients returning to the clinic for their initial post-RFA follow-up visit. At 7-33 weeks, few patients were attending their initial post-RFA follow-up visit, whereas a majority of patients were attending their second follow-up visit. The median (range) time from RFA for this data collection was 20.5 (7-33) weeks. The pain questionnaire and follow-up documentation were reviewed, and average NRS scores were collected. If the duration of relief was clearly documented within the EMR, these data were also included. The primary outcome for statistical analysis was NRS and the change in NRS from baseline at each of the 3 predetermined time points. Data on NRS were summarized using means (standard deviation). Statistical analysis was performed in 2 ways: 1) accounting for data dependencies with repeated measures analysis of variance models to account for multiple RFAs from 10 of 31 individuals; and 2) treating all data as independent and using paired $t$ tests for analysis. Both methods produced very similar statistical results and method 2 will be reported for simplicity of interpretation of the results. Statistically significant changes from baseline were assessed using paired $t$ tests. Treatment success was defined as a change in NRS of $\geq 2$, implementing the minimal clinically important difference (MCID) established for chronic musculoskeletal pain (18). Differences between the 
change in NRS and the number of diagnostic blocks (1 vs. 2) was evaluated using 2-sample t tests. The Pearson correlation coefficients $(95 \%$ confidence interval) between the change in NRS and patient BMI was calculated. Statistical significance was defined as $P<0.05$. All analyses were run using $\mathrm{R}$ version 3.3 (Bell Laboratories, Murray Hill, NJ).

\section{RESULTS}

In the data sample collected from April of 2015 to June of 2017, 33 patients underwent genicular RFA. Out of these 33 individuals, 49 total knees were performed. Of these 49 knees, pre-RFA average pain NRS scores were available on 47 knees from 31 individuals, which were included in the analysis. Baseline demographic data, interventional history, and procedural characteristics are listed in Table 1. The average age was 57.7 years with an average BMI of 34.6. Several patients had undergone prior interventions, with presumed treatment failure. Fourteen $(29.8 \%)$ patients were status post-knee surgery. Forty-one $(87.2 \%)$ patients had undergone a prior injection approach: steroid, synvisc, or orthovisc. In this study, more patients underwent one diagnostic block (29 [61.7\%]) versus 2 diagnostic blocks (18) prior to RFA.

As described in the Methods section, data were analyzed at 3 time points: 2 weeks, $4-6$ weeks, and 7-33 weeks (extended follow-up). At the 2 week mark, data were available for 33 knees. At the 4-6 week mark and 7-33 week mark, data were available on 18 knees, respectively. The lack of data and drop-off is multifactorial. Several patients did not return their 2 week pain questionnaire, and many were lost to follow-up after RFA. If the patient's average pain was not specifically documented in the EMR, these data were not included in the study.

Table 2 lists the NRS data at each time point. The mean pre-RFA baseline NRS for the entire study population ( $n=47$ knees) was 6.4. The documented change in NRS is based off of the pre-RFA baseline NRS for each group at each specific time point. The mean pre-RFA NRS for the 33 knees evaluated at the 2 week mark was 6.8 , which decreased to 3.4 at 2 weeks. This resulted in a change in mean NRS of -3.4 . At $4-6$ weeks, the decrease in mean NRS
Table 1. Baseline demographics, interventional history, and procedural characteristics.

\begin{tabular}{|l|l|}
\hline $\mathrm{n}$ (knees) & 47 \\
\hline Age & $57.7(15.9)$ \\
\hline Gender - female & $24(51.1 \%)$ \\
\hline BMI & $34.6(9.3)$ \\
\hline Side - left & $20(42.6 \%)$ \\
\hline Blocks prior to RFA & \\
\hline 1 & $29(61.7 \%)$ \\
\hline 2 & $18(38.3 \%)$ \\
\hline Prior knee surgery & $14(29.8 \%)$ \\
\hline Prior total knee arthroplasty & $1(2.1 \%)$ \\
\hline Prior injection & \\
\hline Steroid & $11(23.4 \%)$ \\
\hline Synvisc & $15(31.9 \%)$ \\
\hline Steroid and synvisc & $14(29.8 \%)$ \\
\hline Orthovisc & $1(2.1 \%)$ \\
\hline None & $6(12.8 \%)$ \\
\hline
\end{tabular}

Data reported as mean (standard deviation) or $\mathrm{n}(\%)$.

Table 2. NRS data.

\begin{tabular}{|l|c|c|c|c|}
\hline & $\begin{array}{c}\text { Pre-RFA } \\
(\mathbf{n}=\mathbf{4 7})\end{array}$ & $\begin{array}{c}\text { Post 2 } \\
\text { Weeks } \\
(\mathbf{n}=\mathbf{3 3})\end{array}$ & $\begin{array}{c}\text { Post 4-6 } \\
\text { Weeks } \\
(\mathbf{n}=\mathbf{1 8})\end{array}$ & $\begin{array}{c}\text { Extended } \\
\text { Follow- } \\
\text { Up* } \\
(\mathbf{n}=\mathbf{1 8})\end{array}$ \\
\hline NRS & $6.4(1.9)$ & $3.4(3.0)$ & $2.9(2.6)$ & $5.1(2.8)$ \\
\hline $\begin{array}{l}\text { Change from } \\
\text { baseline }\end{array}$ & & $-3.4(3.1)$ & $-3.6(2.0)$ & $-1.8(2.6)$ \\
\hline$P$ value & & $<0.001$ & $<0.001$ & 0.009 \\
\hline
\end{tabular}

Data reported as mean (standard deviation).

*Median final follow-up was 20.5 weeks with a range of 7-33 weeks.

was -3.6 , and at 7-33 weeks, the decrease was -1.8 . Stated differently, the mean NRS pain score decreased by $50 \%$ at 2 weeks $(-3.4 ; P<0.001)$, $55 \%$ at $4-6$ weeks $(-3.6 ; P<0.001)$, and $26 \%$ at $7-33$ weeks $(-1.8 ; P=0.009)$. A total of 8 patients $(12$ knees) provided specific documentation regarding the total duration of relief following RFA. The mean duration was 39 weeks or approximately 9 months. There was high variability in duration, ranging from 3-52 weeks with a median of 27.5 .

Outcomes were compared between individuals who received 1 versus 2 diagnostic genicular nerve blocks prior to RFA (Table 3). There were no statistically significant differences between these groups at 2 weeks 
Table 3. Number of diagnostic blocks.

\begin{tabular}{|l|c|c|c|}
\hline & $\begin{array}{c}\text { Post 2 } \\
\text { Weeks } \\
(\mathbf{n}=\mathbf{3 3})\end{array}$ & $\begin{array}{c}\text { Post 4-6 } \\
\text { Weeks } \\
(\mathbf{n}=\mathbf{1 8})\end{array}$ & $\begin{array}{c}\text { Extended } \\
\text { Follow-Up* } \\
(\mathbf{n}=\mathbf{1 8})\end{array}$ \\
\hline 1 Block & $-4.0(2.6)$ & $-3.5(2.7)$ & $-3.1(2.2)$ \\
\hline 2 Blocks & $-2.4(3.8)$ & $-3.6(0.9)$ & $+0.1(2.1)$ \\
\hline$P$ value & 0.189 & 0.893 & 0.008 \\
\hline
\end{tabular}

Data reported as mean (standard deviation)

*Median final follow-up was 20.5 weeks with a range of 7-33 weeks.

Table 4. BMI.

\begin{tabular}{|c|c|c|}
\hline $\begin{array}{c}\text { Post } 2 \text { Weeks } \\
(\mathbf{n}=\mathbf{3 3})\end{array}$ & $\begin{array}{c}\text { Post 4-6 Weeks } \\
(\mathbf{n}=\mathbf{1 8})\end{array}$ & $\begin{array}{c}\text { Extended Follow-Up* } \\
(\mathbf{n}=\mathbf{1 8})\end{array}$ \\
\hline $0.18(-0.18,0.49)$ & $0.29(-0.20,0.67)$ & $0.46(-0.01,0.77)$ \\
\hline
\end{tabular}

Data reported as correlation coefficients ( $95 \%$ confidence interval, $\mathrm{P}$ value).

*Median final follow-up was 20.5 weeks with a range of 7-33 weeks.

or 4-6 weeks post-RFA. At extended follow-up, those who received 1 block had a decrease in mean NRS of -3.1 , whereas those who received 2 blocks had a slight increase in mean NRS of +0.1 . This difference achieved statistical significance $(P=0.008)$, and the time post-RFA was not different between groups $(P=$ $0.413)$. There was no correlation identified between $\mathrm{BMI}$ and change in NRS at any time point (Table 4).

There were no reported postprocedural adverse events in this study. This includes post-RFA paresthesias, neuropathic pain, infection, weakness, or hematoma.

\section{DISCUSSION}

This study investigated the treatment effectiveness of C-RFA of the genicular nerves on chronic knee pain in the setting of OA. There is limited literature available on the efficacy of genicular C-RFA in this patient population, and it has yet to be compared side-by-side with conventional or pulsed RFA (19). In this study, C-RFA of the genicular nerves lead to clinically significant pain relief at 2 weeks $(n=33)$ and $4-6$ weeks $(n=18)$ postintervention. There was statistically successful pain relief at $7-33$ weeks $(n=18)$ with a change in mean NRS of -1.8 , but this did not meet the established MCID cutoff defined as a decrease in NRS of 2. Regarding duration of therapeutic effect, only 8 patients ( 12 knees) were included. Documentation must contain one of the following statements, for example: "pain relief lasted," or "duration of effect," et cetera. The mean duration of treatment effect was 39 weeks, or approximately 9 months (3-52 weeks). The high variability and small $\mathrm{N}$ does limit the generalizability of this information. Santana Pineda et al (20) recently published a prospective observational study using ultrasound guidance and conventional RFA. In this study, patients achieved a $32 \%$ reduction in the visual analog scale at 12 months postprocedure (20). lannaccone et al (21) studied pain relief at 6 months post-C-RFA, with treatment responders achieving a $64 \%$ reduction in average pain using the NRS. We did not achieve the same effectiveness at 7-33 weeks (median 20.5) when comparing with these studies. We postulate that more treatment nonresponders may have been captured at the extended follow-up visit. Those who achieved a successful therapeutic response to treatment may be less likely to attend their follow-up appointment. In a cross-sectional study by McCormick et al (22), a 50\% success rate at 6 months postprocedure was achieved when success was defined using the MCID for chronic pain. Using a similar definition, we also achieved a $50 \%$ success rate with 9 out of 18 knees reaching the MCID at 7-33 weeks.

A subgroup analysis was performed to evaluate if a relationship existed between $\mathrm{BMI}$ and the primary outcome measure. We hypothesized that those with higher BMI may have worse outcomes, but this correlation was not established. It should also be noted that the average BMI of this patient population was higher than that of previous studies at $34.6(14,20-$ 23). We also hypothesized that outcomes would be better for those individuals who received 2 versus 1 successful diagnostic block(s). By achieving a series of 2 successful blocks, placebo responders may be filtered out (24). There were no statistically significant differences between these groups at 2 weeks or 4-6 weeks. Interestingly, at 7-33 weeks, those who received 1 diagnostic block had better outcomes than those who received $2(P<0.008)$. This places the use of 2 blocks into question, as this does require a separate patient encounter and adds to health care costs. A recent study also demonstrated that a single diagnostic genicular nerve block did not improve the rate of treatment success when compared to no diagnostic block (25). 


\section{CONCLUSIONS}

Our study has several limitations, beginning with its retrospective design and relatively small sample size. This design inherently leads to a higher risk of selection bias. The primary outcome measure for this study was the change in mean NRS pain score, and the mean of ordinal data with a nonnormal distribution lacks validity in statistical analysis. Using the NRS, we defined success as the MCID for chronic musculoskeletal pain, which serves as a weak measure of clinical efficacy (18). However, this study did reveal a decrease in mean NRS of -3.6 at 4-6 weeks and would have stood up to more stringent criterion. The authors targeted 3 genicular nerves supplying the anterior knee. Previous studies have identified 4 accessible articular branches, including the middle branch located superior to the patella (15). This articular branch was excluded for patient comfort, and the effect this may have had on the treatment response is unclear. This study would be strengthened by the use of self-reported functional status measures, psychosocial factors, and mood disturbance as they play an integral role in chronic pain (26). There was also significant loss to follow-up. The attrition rate was $63 \%$ at $4-6$ weeks and $7-33$ weeks, which may affect the generalizability of the results.

Genicular C-RFA did demonstrate successful reduction in chronic knee pain secondary to OA up to 4-6 weeks postprocedure. Statistically significant but less robust improvements in pain occurred at 7-33 weeks (median 20.5 weeks). Further prospective studies are necessary to determine the effectiveness and durability of genicular C-RFA side-by-side with common injection approaches, such as HA-based injections. Continued efforts will also help to establish the most successful procedural approach and patient selection factors to predict success. 


\section{REFERENCES}

1. Altman R, Asch E, Bloch D, Bole G, Borenstein D, Brandt K, Christy W, Cooke TD, Greenwald R, Hochberg M, Howell D, Kaplan D, Koopman W, Longley S, Mankin H, McShane DJ, Medsger T, Meenan R, Mikkelsen W, Moskowitz R, Murphy W, Rothschild B, Segal M, Sokoloff L, Worlfe F. Development of criteria for the classification and reporting of osteoarthritis. Classification of osteoarthritis of the knee. Diagnostic and Therapeutic Criteria Committee of the American Rheumatism Association. Arthritis Rheum 1986; 29:1039-1049.

2. Kraus VB, Blanco FJ, Englund M, Karsdal MA, Lohmander LS. Call for standardized definitions of osteoarthritis and risk stratification for clinical trials and clinical use. Osteoarthritis Cartilage 2015; 23:1233-1241.

3. Barbour KE, Helmick CG, Boring M, Brady TJ. Vital signs: Prevalence of doctor-diagnosed arthritis and arthritis-attributable activity limitation: United States, 2013-2015. MMWR Morb Mortal Wkly Rep 2017; 66:246-253.

4. Helmick CG, Felson DT, Lawrence RC, Gabriel S, Hirsch R, Kwoh CK, Liang MH, Kremers HM, Mayes MD, Merkel PA, Pillemer SR, Reveille JD, Stone JH. Estimates of the prevalence of arthritis and other rheumatic conditions in the United States. Part I. Arthritis Rheum 2008; 58:5-25.

5. Jafarzadeh SR, Felson DT. Updated estimates suggest a much higher prevalence of arthritis in United States adults than previous ones. Arthritis Rheum 2018; 70:185-192.

6. Cross M, Smith E, Hoy D, Nolte S, Ackerman I, Fransen M, Bridgett L, Williams S, Guillemin F, Hill CL, Laslett LL, Jones G, Cicuttini F, Osborne R, Vos T, Buchbinder R, Woolf A, March L. The global burden of hip and knee osteoarthritis: Estimates from the global burden of disease 2010 study. Ann Rheum Dis 2014; 73:1323-1330

7. Nguyen US, Zhang Y, Zhu Y, Niu J, Zhang B, Felson DT. Increasing prevalence of knee pain and symptomatic knee osteoarthritis: Survey and cohort data. Ann Intern Med 2011; 155:725732.

8. Bernstein J, Derman P. Dramatic increase in total knee replacement utilization rates cannot be fully explained by a disproportionate increase among younger patients. Orthopedics 2014; 37:656-659.

9. Bastick AN, Runhaar J, Belo JN, Bierma-Zeinstra SM. Prognostic factors for progression of clinical osteoarthritis of the knee: A systematic review of observational studies. Arthritis Res Ther 2015; 17:1-13.

10. Gallagher B, Tjoumakaris FP, Harwood MI, Good RP, Ciccotti MG, Freedman KB. Chondroprotection and the prevention of osteoarthritis progression of the knee: A systematic review of treatment agents. Am J Sports Med 2015; 43:34-44.

11. He WW, Kuang MJ, Zhao J, Sun L, Lu B, Wang Y, Ma JX, Ma $\mathrm{XL}$. Efficacy and safety of intraarticular hyaluronic acid and corticosteroid for knee osteoarthritis: A meta-analysis. Int J Surg 2017; 39:95-103.

12. McAlindon TE, LaValley MP, Harvey WF, Price LL, Driban JB, Zhang M, Ward RJ. Effect of intra-articular triamcinolone vs. saline on knee cartilage volume and pain in patients with knee osteoarthritis: A randomized clinical trial. JAMA 2017; 317:19671975.

13. Laudy AB, Bakker EW, Rekers M, Moen MH. Efficacy of plateletrich plasma injections in osteoarthritis of the knee: A systematic review and meta-analysis. Br J Sports Med 2015; 49:657-672.

14. Choi WJ, Hwang SJ, Song JG, Leem JG, Kang YU, Park PH,
Shin JW. Radiofrequency treatment relieves chronic knee osteoarthritis pain: A double-blind randomized controlled trial. Pain 2011; 152:481-487.

15. Franco CD, Buvanendran A, Petersohn JD, Menzies RD, Menzies LP. Innervation of the anterior capsule of the human knee: Implications for radiofrequency ablation. Reg Anesth Pain Med 2015; 40:63-68.

16. Rojhani S, Qureshi Z, Chhatre A. Water-cooled radiofrequency provides pain relief, decreases disability, and improves quality of life in chronic knee osteoarthritis. Am J Phys Med Rehabil 2017; 96:e5-e8.

17. Roy C, Chatterjee N, Ganguly S, Sengupta R. Efficacy of combined treatment with medial branch radiofrequency neurotomy and steroid block in lumbar facet joint arthropathy. J Vasc Interv Radiol 2012; 23:1659-1664

18. Salaffi F, Stancati A, Silvestri CA, Ciapetti A, Grassi W. Minimal clinically important changes in chronic musculoskeletal pain intensity measured on a numerical rating scale. Eur J Pain 2004; 8:283-291.

19. Gupta A, Huettner DP, Dukewich M. Comparative effectiveness review of cooled versus pulsed radiofrequency ablation for the treatment of knee osteoarthritis: A systematic review. Pain Physician 2017; 20:155-171.

20. Santana Pineda MM, Vanlinthout LE, Moreno Martin A, Van Zundert J, Rodriguez Huertas F, Novalbos Ruiz JP. Analgesic effect and functional improvement caused by radiofrequency treatment of genicular nerves in patients with advanced osteoarthritis of the knee until 1 year following treatment. Reg Anesth Pain Med 2017; 42:62-68.

21. lannaccone $F$, Dixon S, Kaufman A. A review of long-term pain relief after genicular nerve radiofrequency ablation in chronic knee osteoarthritis. Pain Physician 2017; 20:437-444.

22. McCormick ZL, Korn M, Reddy R, Marcolina A, Dayanim D, Mattie R, Cushman D, Bhave M, McCarthy RJ, Khan D, Nagpal $G$, Walega DR. Cooled radiofrequency ablation of the genicular nerves for chronic pain due to knee osteoarthritis: Six-month outcomes. Pain Med 2017; 18:1631-1641.

23. Sari S, Aydin ON, Turan Y, Ozlulerden P, Efe U, Kurt Omurlu I. Which one is more effective for the clinical treatment of chronic pain in knee osteoarthritis: Radiofrequency neurotomy of the genicular nerves or intra-articular injection? Int J Rheum Dis 2018; 21:1772-1778.

24. Derby R, Melnik I, Lee JE, Lee SH. Correlation of lumbar medial branch neurotomy results with diagnostic medial branch block cutoff values to optimize therapeutic outcome. Pain Med 2012; 13:1533-1546.

25. McCormick ZL, Reddy R, Korn M, Dayanim D, Syed RH, Bhave M, Zhukalin M, Choxi S, Ebrahimi A, Kendall MC, McCarthy RJ, Khan D, Nagpal G, Bouffard K, Walega DR. A prospective randomized trial of prognostic genicular nerve blocks to determine predictive value for the outcome of cooled radiofrequency ablation for chronic knee pain due to osteoarthritis. Pain Med 2018; 19:1628-1638.

26. Deyo RA, Dworkin SF, Amtmann D, Andersson G, Borenstein D, Carragee E, Carrino J, Chou R, Cook K, DeLitto A, Goertz C, Khalsa P, Loeser J, Mackey S, Panagis J, Rainville J, Tosteson R, Turk D, Van Korff M, Weiner DK. Report of the NIH Task Force on research standards for chronic low back pain. Pain Med 2014; 15:1249-1267. 